\title{
Correction to: New Developments on Computational Methods and Imaging in Biomechanics and Biomedical Engineering
}

João Manuel R. S. Tavares and Paulo Rui Fernandes

\section{Correction to:}

J. M. R. S. Tavares and P. R. Fernandes (eds.), New Developments on Computational Methods and Imaging in Biomechanics and Biomedical Engineering, Lecture Notes in Computational Vision and Biomechanics 33, https://doi.org/10.1007/978-3-030-23073-9

In the original publication of this book, the volume number was incorrect. It should read "33" instead of "999". This has now been corrected. 